\title{
Ixazomib-based frontline therapy in patients with newly diagnosed multiple myeloma in real-life practice showed comparable efficacy and safety profile with those reported in clinical trial: a multi-center study
}

\author{
Jing $\mathrm{Li}^{1} \cdot \mathrm{Li} \mathrm{Bao}^{2} \cdot$ Zhongjun Xia $^{3} \cdot$ Sili Wang ${ }^{4} \cdot \mathrm{Xin} \mathrm{Zhou}^{5} \cdot \mathrm{Kaiyang} \mathrm{Ding}^{6} \cdot$ Wenhao Zhang $^{7} \cdot$ Wei Yang $^{8} \cdot$ \\ Bingzong $\mathrm{Li}^{9} \cdot$ Chengcheng Fu $^{10} \cdot$ Bing Chen $^{11}$. Luoming Hua ${ }^{12}$. Liang Wang ${ }^{13}$. Jun Luo ${ }^{14} \cdot$ Yang Yang $^{1}$.

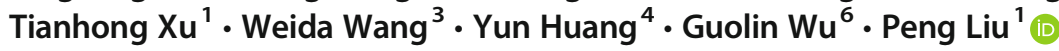

Received: 9 June 2020 / Accepted: 24 August 2020 / Published online: 6 September 2020

(C) The Author(s) 2020

\begin{abstract}
The induction therapy containing ixazomib, an oral proteasome inhibitor, has shown favorable efficacy and safety in clinical trials, but its experience in real-life remains limited. In routine practice, few patients received ixazomib-based induction therapy due to reasons including (1) patients' preference on oral regimens, (2) concerns on adverse events (AEs) of other intravenous/subcutaneous regimens, (3) requirements for less center visits, and (4) fears of COVID-19 and other infectious disease exposures. With the aim of assessing the real-life effectiveness and safety of ixazomib-based induction therapy, we performed this multi-center, observational study on 85 newly diagnosed multiple myeloma (NDMM) patients from 14 medical centers. Ixazomib-based regimens included ixazomib-lenalidomide-dexamethasone (IRd) in $44.7 \%$ of patients, ixazomib-dexamethasone (Id) in $29.4 \%$, and Id plus another agent (doxorubicin, cyclophosphamide, thalidomide, or daratumumab) in 25.9\%. Different ixazomib-based therapies were applied due to (1) financial burdens or limitations on local health insurance coverage, (2) concerns on treatment tolerance, and (3) drug accessibility issue. Ten patients received ixazomib maintenance. The median age was 67 years; $43.5 \%$ had ISS stage III disease; 48.2\% had an Eastern Cooperative Oncology Group performance score $\geq 2$; and $17.6 \%$ with high-risk cytogenetic abnormalities. Overall response rate for all 85 patients was $95.3 \%$, including $65.9 \%$ very good partial response or better and $29.5 \%$ complete responses. The median time to response was 30 days. The response rate was similar across different ixazomib-based regimens. Median progression-free survival was not reached. Severe AEs ( $\geq$ grade 3) were reported in $29.4 \%$ of patients. No grade $3 / 4$ peripheral neuropathy (PN) occurred. Patients received a median of 6 (range 1-20) cycles of ixazomib treatment; 56.6\% remained on treatment at data cutoff; $15.3 \%$ discontinued treatment due to intolerable AEs. These results support that the ixazomib-based
\end{abstract}

Jing Li, Li Bao and Zhongjun Xia contributed equally to this work.

Peng Liu

liu.peng@ zs-hospital.sh.cn

1 Department of Hematology, Zhongshan Hospital, Fudan University, 180 Fenglin Road, Shanghai 200032, People's Republic of China

2 Department of Hematology, Beijing Jishuitan Hospital, Beijing, China

3 Department of Hematologic Oncology, Sun Yat-sen University Cancer Center, Guangzhou, China

4 The First Affiliated Hospital of Xiamen University, Xiamen, China

5 Wuxi People Hospital, WuXi, China

6 Anhui Provincial Cancer Hospital, Hefei, China

7 Xinhua Hospital Affiliated to Shanghai Jiaotong University School of Medicine, Shanghai, China
8 Shengjing Hospital of China Medical University, Shengjing, China

9 The Second Affiliated Hospital of Soochow University, Suzhou, Jiangsu, China

10 Department of Hematology, The First Affiliated Hospital of Soochow University, Jiangsu Institute of Hematology, Suzhou, China

11 Department of Hematology, The Affiliated Nanjing Drum Tower Hospital, Nanjing University Medical School, Nanjing, China

12 The Affiliated Hospital of Hebei University, Baoding, China

13 Department of Hematology, ZhuJiang Hospital of Southern Medical Univeristy, Guangzhou, China

14 The First Affiliated Hospital of Guangxi Medical University, Nanning, Guangxi, China 
frontline therapy was highly effective with acceptable toxicity in routine practice and the ixazomib oral regimens could be good alternative options for NDMM patients.

Keywords Myeloma $\cdot$ Frontline $\cdot$ Ixazomib $\cdot$ Real-world

\section{Introduction}

Substantial improvement in outcomes of multiple myeloma (MM) has been observed over the past decades [1], and the treatment paradigm for MM has been considerably evolved [2]. For newly diagnosed MM (NDMM), induction therapy with multi-agents combination has become a standard of care, with numeral phase 3 trials showing superior survival benefit using triplet or quadruplet regimens containing proteasome inhibitor bortezomib [3-5]. Besides, the concept of continuous or long-term therapy in MM has been widely accepted, since maintenance therapy or continued initial therapy demonstrated obvious prolonged survival $[6,7]$. In this context, the proteasome inhibitor bortezomib has become one of the backbone agents in the frontline treatment for MM; however, the non-oral administration and concerns in side effects including peripheral neuropathy hampered its long-term use in the real-world setting, with a median duration of treatment of only 6 months for bortezomib-based frontline therapy [8].

Ixazomib is the first oral proteasome inhibitor approved for the treatment in MM patients who have received at least one prior therapy in over 60 countries. The all-oral combination of weekly ixazomib plus lenalidomide and dexamethasone (IRd) has demonstrated durable efficacy and well-tolerated toxicities in phase 3 trial TOURMALINE-MM1 $[9,10]$ and in real-life practice [11].

The all-oral triplet regimen of IRd exhibited favorable efficacy with an acceptable toxicity profile in NDMM, according to the results of a phase $1 / 2$ study (NCT01217957) [12, 13], with an overall response rate (ORR) of $92 \%$. Besides, the ixazomib-based all-oral regimen combined with cyclophosphamide and dexamethasone (ICd) has shown promising efficacy in phase 2 studies $[14,15]$. Furthermore, all-oral regimens, including IRd combination, have been suggested as possible options during the pandemic of COVID-19 in different consensus or opinions [16-19], owing to the fact that using an oral regimen may help to reduce the risk for virus exposure and infection. However, effectiveness and tolerability of novel agent for MM treatment in routine clinical practice often differ from the results reported in registered clinical trials $[8,20,21]$, and the published data on frontline treatment with ixazomibbased regimes in large phase 3 trials and in the real-world setting are limited. Herein, we report the real-world data of the initial effectiveness and safety profile of ixazomib-based frontline therapy in patients with NDMM from 14 centers in China, in hopes of providing informative data to help therapeutic decision-making.

\section{Patients and methods}

This is a national, multi-centric, retrospective, observational study analyzing data collected from 85 patients with NDMM, who received ixazomib-containing regimen as induction therapy in real-life routine practice. Patients were treated at 14 different medical centers in China between August 2018 and April 2020, since ixazomib was officially approved by the China Food and Drug Administration in April 2018.

For inclusion in the present study, patients had to be diagnosed with MM by the 2014 criteria of International Myeloma Working Group (IMWG) [22] and previously untreated before receiving at least one cycle of ixazomib therapy. The records including demographics, disease characteristics, molecular cytogenetic subtype, treatment exposure, treatment response, treatment duration, adverse events (AEs), and survival outcome were retrieved and analyzed.

The main objectives of this analysis were to determine the ORR (partial response (PR) or better) and the proportion of patients with CR or very good PR (VGPR) of frontline ixazomibbased regimens in real-life setting. Secondary endpoints included estimation of progression-free survival (PFS), overall survival (OS), and the response rate within patient subgroups categorized by cytogenetic risk stratification defined by fluorescence in situ hybridization (FISH) [23] or metaphase cytogenetics. High-risk cytogenetic abnormalities (CA) included del 17, $t(4 ; 14), t(14 ; 16)$.

The other primary objective of this study was to assess the safety profile of frontline ixazomib therapy, including the occurrence rate of common AEs and severe AEs, the duration of ixazomib treatment exposure, and the rate and reasons for drug discontinuation.

The study was approved by the institutional ethics committee (B2019-228R) and conducted in accordance with the 1964 Helsinki declaration and its later amendments. Patient care and evaluation were determined by their own treating physicians and effected by the patients' own preference.

All 85 patients with symptomatic MM received at least one ixazomib-based therapy. Ixazomib-containing regimens analyzed in this study were listed as follows: IRd regimen (ixazomib $4 \mathrm{mg}$ on days 1,8 , and 15 , lenalidomide $25 \mathrm{mg}$ on days $1-21$, dexamethasone $40 \mathrm{mg}$ weekly, every 28 days), Id regimen (ixazomib $4 \mathrm{mg}$ on days 1,8 , and 15 , dexamethasone $40 \mathrm{mg}$ weekly, every 28 days), and Id regimen in combination with other chemotherapeutic agents or monoclonal antibody (ITd, Id plus daily thalidomide $100 \mathrm{mg}$; ICd, Id plus weekly oral cyclophosphamide $300 \mathrm{mg} / \mathrm{m}^{2}$; IAd, Id plus doxorubicin $9 \mathrm{mg} / \mathrm{m}^{2}$ IV d1-4; DId, Id plus daratumumab administered IV 
Table 1 Baseline clinical characteristics of the patients included in the analysis

\begin{tabular}{|c|c|}
\hline Characteristic & All Patients $(N=85)$ \\
\hline Median age (range) & $67(35-87)$ \\
\hline \multicolumn{2}{|l|}{ Age group (\%) } \\
\hline $18-64$ years & $41(48.2)$ \\
\hline $65-74$ years & $26(30.6)$ \\
\hline$\geq 75$ years & $18(21.1)$ \\
\hline Male, $n(\%)$ & $48(56.5)$ \\
\hline \multicolumn{2}{|l|}{ ISS stage at diagnosis, $n(\%)$} \\
\hline I & $22(25.9)$ \\
\hline II & $26(30.6)$ \\
\hline III & $37(43.5)$ \\
\hline High-risk cytogenetic abnormalities ${ }^{\mathrm{a}, \mathrm{b}}(\%)$ & $15(17.6)$ \\
\hline Del 17 & $4(5.9)$ \\
\hline$t(4 ; 14)$ & $11(16.2)$ \\
\hline$t(14 ; 16)$ & $4(5.9)$ \\
\hline \multicolumn{2}{|l|}{ ECOG performance status, $n(\%)$} \\
\hline $0-1$ & $44(51.8)$ \\
\hline 2 & $20(23.5)$ \\
\hline $3-4$ & $21(24.7)$ \\
\hline Serum creatinine $>177 \mu \mathrm{mol} / \mathrm{L}, n(\%)$ & $18(21.2)$ \\
\hline Extramedullary disease & $6(7.1)$ \\
\hline Eligibility for phase $\mathrm{I} / \mathrm{II}$ trial $^{\mathfrak{c}}$ & $30(35.3)$ \\
\hline \multicolumn{2}{|l|}{ Regimens, $n(\%)$} \\
\hline Ixazomib plus lenalidomide-dexamethasone (IRd) & $38(44.7)$ \\
\hline Ixazomib plus dexamethasone (Id) & $25(29.4)$ \\
\hline Id plus chemotherapeutics/monoclonal antibody ${ }^{\mathrm{d}}$ & $22(25.9)$ \\
\hline
\end{tabular}

ISS, International Staging System

${ }^{a}$ High-risk cytogenetic abnormalities were detected by fluorescence in situ hybridization (FISH) or metaphase cytogenetics, including del $17, t(4 ; 14), t(14 ; 16)$

${ }^{\mathrm{b}} 17$ patients with no FISH data were not included

${ }^{\mathrm{c}}$ Patients whose baseline characteristics theoretically fulfilled the in- and exclusion criteria for the phase $1 / 2$ study (NCT01217957, a study evaluated the safety and efficacy of IRd regimen, followed by single-agent ixazomib maintenance, in patients with newly diagnosed myeloma) were defined as eligible for phase I/II trial

${ }^{\mathrm{d}}$ This subgroup included Id plus other chemotherapeutics or monoclonal antibody: 11 cases with doxorubicin, 7 with cyclophosphamide, 3 with thalidomide, and 1 with daratumumab at $16 \mathrm{mg} / \mathrm{kg}$ weekly for 8 weeks, followed by every other week for 8 doses, and then every 4 weeks). Selected patients received long-term single-agent ixazomib maintenance after up to 12 cycles of ixazomib-based induction therapy. Patients received continuous ixazomib-based treatment until disease progression, intolerable toxicity, or patient/physician decision to end treatment. Dose modifications of studying drugs for toxic effects were recorded. Interruption of treatment for stem cell collection or stem cell transplantation (SCT) was allowed.

Response assessment and safety evaluation were done in patients every month according to the 2016 IMWG response criteria [24]. A bone marrow aspiration/biopsy was required to confirm a complete response (CR).

Statistical analysis was conducted using SPSS statistics software (version 26, IBM, Armonk, NY). Statistical significance was set at a two-sided value of $P<0.05$. Descriptive statistics were used to describe baseline characteristics, response, and AEs data. The Chi-square method or Fisher's exact test was used to determine differences between nominal variables. Survival curves were calculated using the Kaplan-Meier curves, and log-rank tests were performed to assess the differences between the groups.

\section{Results}

\section{Patients}

A total of 85 patients with NDMM from 14 medical centers treated with at least one cycle of ixazomib-based 
Table 2 Treatment exposure in all patients and patients who received maintenance

\begin{tabular}{|c|c|c|}
\hline & All patients $(N=85)$ & $\begin{array}{l}\text { Patients who received ixazomib } \\
\text { maintenance }(N=10)\end{array}$ \\
\hline Median cycles of ixazomib received, $n$ (range) & $6(1-20)$ & $14(11-20)$ \\
\hline \multicolumn{3}{|l|}{ Cycles of ixazomib received, $n(\%)$} \\
\hline$\geq 3$ & $74(87.1)$ & $10(100.0)$ \\
\hline$\geq 6$ & $51(60.0)$ & $10(100.0)$ \\
\hline$\geq 9$ & $24(28.2)$ & $10(100.0)$ \\
\hline Number of patients with dose reduction, $n(\%)$ & $3(3.6)$ & 0 \\
\hline Patients proceeded to SCT & $6(7.1)$ & 0 \\
\hline Patients remaining on treatment, $n(\%)$ & $48(56.5)$ & $9(90.0)$ \\
\hline \multicolumn{3}{|l|}{ Reason for ending treatment, $n(\%)$} \\
\hline Adverse event & $13(15.3)$ & 0 \\
\hline Disease progression & $6(7.1)$ & $1(10.0)$ \\
\hline Another ${ }^{a}$ & $18(21.1)$ & 0 \\
\hline
\end{tabular}

$S C T$, stem cell transplantation

${ }^{\text {a }}$ Reasons included proceeding to SCT, alternate therapy, poor compliance, loss of drug accessibility during the pandemic of COVID-19 and economic concerns regimens as frontline induction therapy were included in this study. Table 1 summarized the patient demographic and disease features at the time of diagnosis. The overall median age at diagnosis was 67 years old (range, 35-87). Almost half $(43.5 \%)$ of patients had an advanced ISS stage III disease, and $48.2 \%$ of patients had an Eastern Cooperative Oncology Group (ECOG) performance score of $\geq 2$. On cytogenetics and FISH analysis (data available in 68 patients), $17.6 \%$ of patients were identified with high-risk CA, including $4(5.9 \%)$ patients with del $17 \mathrm{p}$, $11(16.2 \%)$ with $t(4 ; 14)$, and $4(5.9 \%)$ with $t(14 ; 16)$. Extramedullary lesion was found in $6(7.1 \%)$ patients. On the basis of exclusion criteria of the phase $1 / 2$ study (NCT01217957) evaluating IRd regimen in NDMM [12], 30 (35.3\%) patients were ineligible for this clinical trial. Criteria leading to ineligibility in the clinical trial included ECOG PS of more than $2(70.0 \%)$, grade 2 or higher peripheral neuropathies $(10.0 \%)$, uncontrolled cardiovascular conditions within the past 6 months $(10.0 \%)$, prior malignancy within 2 years $(3.3 \%)$, and others $(6.7 \%)$.

\section{Treatment regimen and exposure}

Ixazomib-based regimens given to all patients were shown in Table 1, including IRd regimen in $38(44.7 \%)$ patients, Id in $25(29.4 \%)$ patients, and Id plus chemotherapeutics/other agents (11 cases with doxorubicin, 7 with cyclophosphamide, 3 with thalidomide, and 1 with daratumumab) in $22(25.9 \%)$. A total of 10 patients received single-agent ixazomib maintenance after median 8 (range, 6-12) cycles of ixazomibcontaining induction therapy (Table 2$)$. Six $(7.1 \%)$ of the patients received SCT after induction therapy with ixazomib.
Treatment exposure for all patients and those who received ixazomib maintenance were documented in Table 2. At data cutoff, the median follow-up time for all patients included was 10.3 months (range, 1.1-22.9). During the follow-up period, the median cycles of ixazomib therapy received by all patients and those who had ixazomib maintenance were 6 (range, 120 ) cycles and 14 (range, 11-20), respectively. At data cutoff, $56.5 \%(48 / 85)$ of patients remained on ixazomib treatment, including $90 \%(9 / 10)$ of those receiving single-agent ixazomib as maintenance. For the remaining $43.5 \%$ of patients who discontinued treatment, the reason for treatment discontinuation (patients could have more than one reason listed) included AEs in $13(15.3 \%)$ patients, disease progression in $6(7.1 \%)$, and other specific reasons in $18(21.1 \%)$. Other reasons included proceeding to SCT, alternate therapy, poor compliance, economic pressure, and loss of drug accessibility during the pandemic of COVID-19.

\section{Response and outcome}

The best confirmed ORR for all 85 patients was $95.3 \%$ (81/ 85 ), including $65.9 \%$ of patients with $\geq$ VGPR and $29.5 \%$ with a CR (including stringent CR) (details are shown in Table 3). The median time to 1 st documented response was 30 days, and the median time to best response was 64 days. The kinetics of response during follow-up are shown in Fig. 1, and the response deepened with time. Among the 15 dataevaluable patients who had high-risk CA, the ORR was $86.7 \%$, including a $33.3 \% \geq \mathrm{VGPR}$ rate and a $13.3 \% \mathrm{CR}$ rate. Although the ORR for high-risk CA subgroup was comparable with the overall population, the $\mathrm{CR}$ rate and $\geq$ VGPR rate were much lower $(p=0.016)$. There was no significant 
Table 3 Treatment outcomes in all patients, those who had high-risk CA, and those who received different ixazomib-based regimens

\begin{tabular}{|c|c|c|c|c|c|}
\hline & $\begin{array}{l}\text { All patients } \\
(N=85)\end{array}$ & $\begin{array}{l}\text { High-risk } \mathrm{CA}^{\mathrm{a}} \\
(n=15)\end{array}$ & $\begin{array}{l}\text { IRd regimen } \\
(n=38)\end{array}$ & $\begin{array}{l}\text { Other triplet regimen }{ }^{\mathrm{b}} \\
(n=22)\end{array}$ & $\begin{array}{l}\text { Id doublet regimen } \\
(n=25)\end{array}$ \\
\hline \multicolumn{6}{|l|}{ Best confirmed response ${ }^{\mathrm{c}}, n(\%)$} \\
\hline ORR ( $\geq$ PR) & $81(95.3)$ & $13(86.7)$ & $35(92.1)$ & $21(95.5)$ & $25(100.0)$ \\
\hline$\geq$ VGPR & $56(65.9)$ & $5(33.3)$ & $22(57.9)$ & $19(86.3)$ & $15(60.0)$ \\
\hline $\mathrm{CR}$ & $25(29.5)$ & $2(13.3)$ & $10(26.3)$ & $7(31.8)$ & $8(32.0)$ \\
\hline PR & $56(65.9)$ & $11(73.3)$ & $25(65.8)$ & $14(63.6)$ & $17(68.0)$ \\
\hline VGPR & $31(36.5)$ & $3(20.0)$ & $12(31.6)$ & $12(54.5)$ & $7(28.0)$ \\
\hline MR & $1(1.2)$ & $1(6.7)$ & $1(2.6)$ & 0 & 0 \\
\hline SD & $3(3.5)$ & $1(6.7)$ & $2(5.3)$ & $1(4.5)$ & 0 \\
\hline $\begin{array}{l}\text { Median time to } 1 \text { st response, } \\
\text { months }\end{array}$ & 1.0 & 1.0 & 1.0 & 1.0 & 0.9 \\
\hline $\begin{array}{l}\text { Median time to best response, } \\
\text { months }\end{array}$ & 2.1 & 1.9 & 2.3 & 2.5 & 1.8 \\
\hline Median PFS, months & $\mathrm{NE}$ & $\mathrm{NE}$ & $\mathrm{NE}$ & $\mathrm{NE}$ & $\mathrm{NE}$ \\
\hline 12-month PFS, \% & 86.3 & 76.6 & 85.5 & 94.7 & 83.8 \\
\hline Median OS, months & NE & $\mathrm{NE}$ & $\mathrm{NE}$ & NE & $\mathrm{NE}$ \\
\hline 12-month OS, \% & 95.3 & 100 & 100 & 95.6 & 89.7 \\
\hline 24-month OS, \% & 84.3 & 66.7 & 66.7 & 95.6 & 89.7 \\
\hline Patients with progression, $n(\%)$ & $11(12.9)$ & $4(26.7)$ & $6(15.8)$ & $1(4.5)$ & $4(16.0)$ \\
\hline
\end{tabular}

$C A$, cytogenetic abnormalities; $O R R$, overall response rate; $P R$, partial response; $V G P R$, very good partial response; $C R$, complete response; $M R$, minimal response; $S D$, stable disease; $P F S$, progression-free survival; $O S$, overall survival; $I R d$, ixazomib plus lenalidomide and dexamethasone; $I d$, ixazomib plus dexamethasone; $N E$, not estimable

${ }^{\text {a }}$ High-risk CA included del $17, t(4 ; 14), t(14 ; 16)$

${ }^{b}$ This subgroup included Id plus another chemotherapeutics or monoclonal antibody: 11 cases with doxorubicin, 7 with cyclophosphamide, 3 with thalidomide, and 1 with daratumumab

${ }^{\mathrm{c}}$ For patients who proceeded to SCT and received further ixazomib maintenance therapy, the best response reported include response post SCT

difference in best confirmed response and time to response among patients who received different ixazomib-based regimens, as shown in Table 3.

OS and PFS for the entire cohort are shown in Fig. 2A. Median OS and median PFS are not estimable (NE) in the whole population and any of the subgroups. At data cutoff, a total of $11(12.9 \%)$ patients experienced disease progression. On landmark analysis, the 12-month PFS and OS for the entire cohort were $86.3 \%$ and $95.3 \%$, respectively. No statistically significant difference was found among patients with different risk stratification in CA (Fig. 2B), those who received different ixazomib-based regimens (Fig. 2C), and patients who were at age of more than 75 years old (Fig. 2D). However, patients who had an ECOG PS > 2 (Fig. 2E) and those who were not eligible for the phase $1 / 2$ trial of IRd in NDMM (NCT01217957) according to its inclusion/exclusion criteria (Fig. 2F) demonstrated inferior PFS $(p<0.05)$ in subgroup analysis.

\section{Safety profile}

Table 4 summarizes the safety profile and common AEs of all patients and those who received different ixazomib-based regimen. Severe adverse events ( $\geq$ grade 3 ) were reported in
$29.4 \%$ patients, including thrombocytopenia ( 7 patients, $8.2 \%$ ), lymphocytopenia ( 3 patients, $3.5 \%$ ), diarrhea (5 patients, $5.9 \%$ ), pneumonia (6 patients, $7.0 \%$ ), hypokalemia (1 patient, $1.7 \%$ ), and severe skin/subcutaneous infection

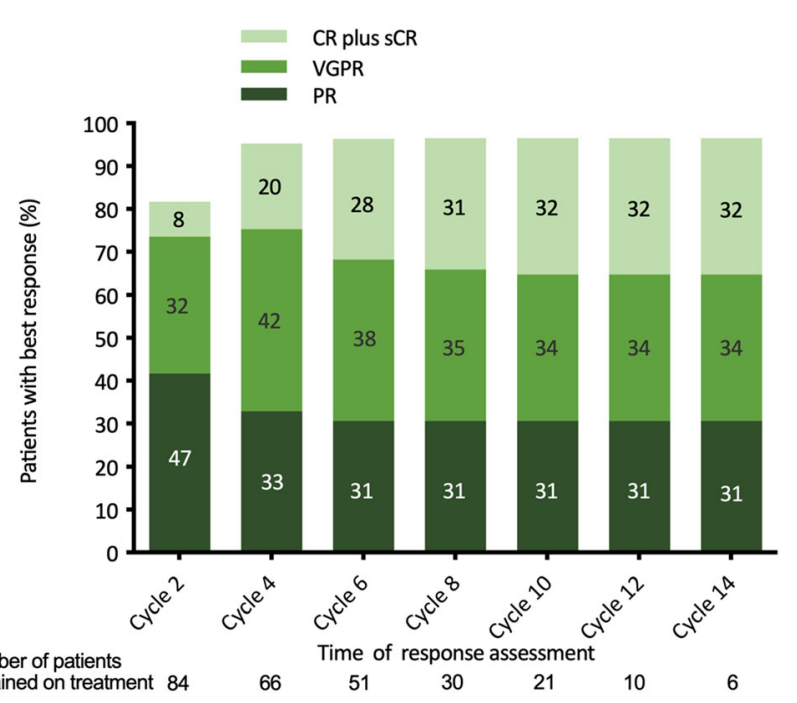

Fig. 1 Changes in response with increasing duration of therapy 

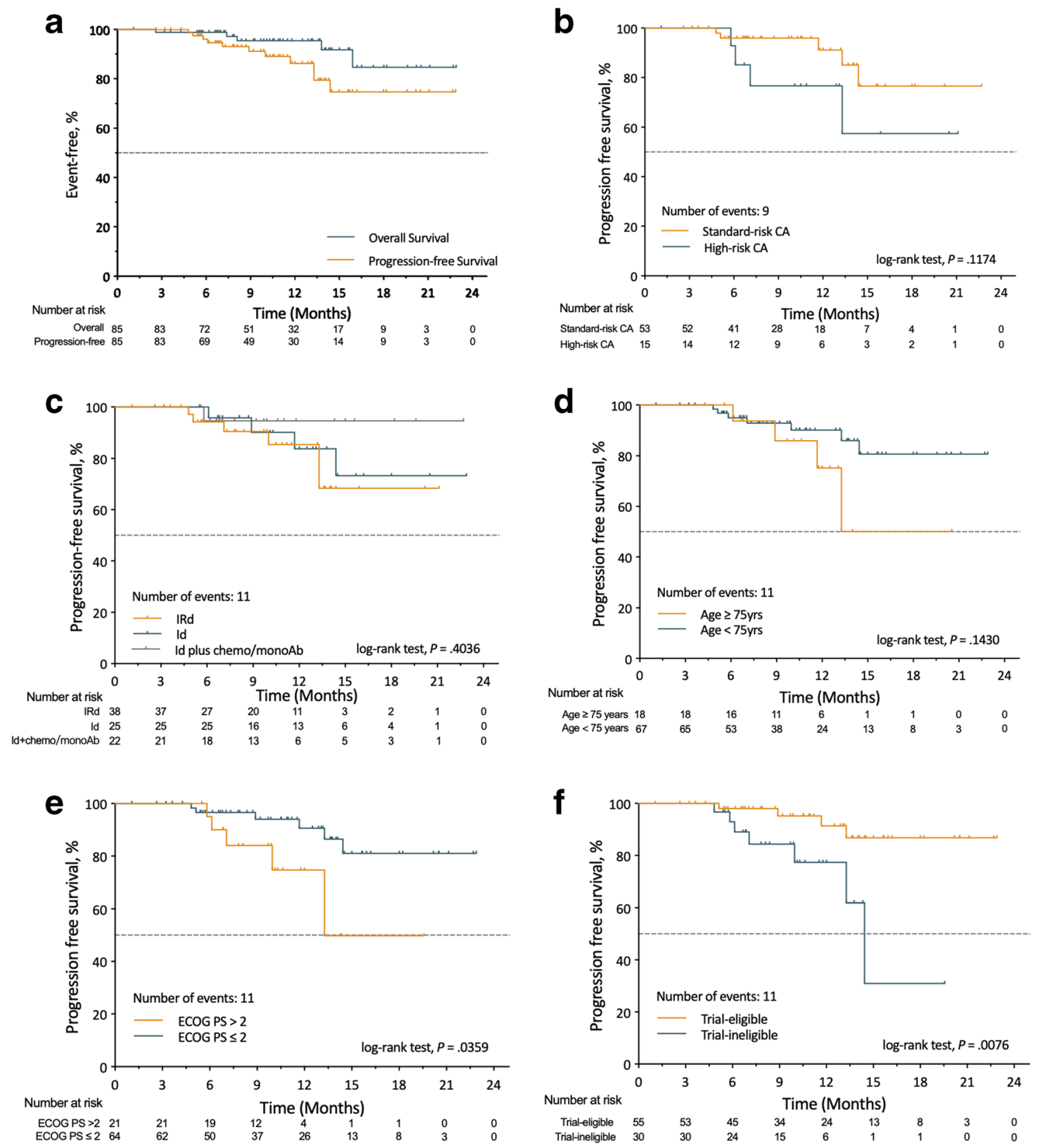

Fig. 2 Kaplan-Meier survival curves. a Overall survival and progressionfree survival (PFS) in all patients; PFS according to risk stratification by cytogenetic abnormalities (b), different ixazomib based regimens (c), age

(2 patients, 2.4\%). Peripheral neuropathy ( $\mathrm{PN}$ ) was reported in 5 patients, but there was no grade $3 / 4$ peripheral neuropathy (PN) occurred. However, we believed some cases with mild PN might have been miss-recorded due to the retrospective nature of this analysis.

Among the 13 patients who had AEs listed as one of the reasons for drug discontinuation, 9 patients endured at least one grade 3/4 AEs, including 4 who had infection, 3 who had diarrhea, 1 who had thrombocytopenia, and 1 who had abdominal distention.

(d), ECOG performance status (e), and trial eligibility (f) based on the criteria of a phase $1 / 2$ study evaluated the weekly oral ixazomib combined with Rd in patients with newly diagnosed myeloma (NCT01217957)

\section{Discussion}

This multi-center, retrospective, observational study highlights the real-world efficacy, feasibility, and tolerability of ixazomib-based frontline therapy in patients with NDMM.

Importantly, the results of this analysis are comparable with those of the phase $1 / 2$ clinical trial of IRd in NDMM (NCT01217957) [12, 13].

Findings from our real-world cohort reflected the outcome of a broader general patient population, which was older than 
Table 4 Safety profile and common AEs of all patients and those who received different ixazomib-based regimen

\begin{tabular}{|c|c|c|c|c|}
\hline & $\begin{array}{l}\text { All patients } \\
(N=85)\end{array}$ & $\begin{array}{l}\text { IRd regimen } \\
(n=38)\end{array}$ & $\begin{array}{l}\text { Other triplet regimen }{ }^{\mathrm{a}} \\
(n=22)\end{array}$ & $\begin{array}{l}\text { Id doublet regimen } \\
(n=25)\end{array}$ \\
\hline \multicolumn{5}{|l|}{ Overall safety profile, $n(\%)$} \\
\hline Any grade $\geq 3 \mathrm{AE}$ & $25(29.4)$ & $9(23.7)$ & $7(31.8)$ & $9(36.0)$ \\
\hline AE leading to discontinuation of ixazomib & $12(14.1)$ & $5(13.2)$ & $3(13.6)$ & $4(16.0)$ \\
\hline AE leading to dose reduction of ixazomib & $3(3.5)$ & $1(2.6)$ & $2(9.1)$ & 0 \\
\hline \multicolumn{5}{|l|}{ Common hematologic AEs, $n(\%)$} \\
\hline Neutropenia & $24(28.2)$ & $11(28.9)$ & $8(36.4)$ & $5(20.0)$ \\
\hline Thrombocytopenia & $24(28.2)$ & $7(18.4)$ & $8(36.4)$ & $9(36.0)$ \\
\hline Anemia & $27(31.8)$ & $12(31.6)$ & $8(36.4)$ & $10(40.0)$ \\
\hline Lymphocytopenia & $29(34.1)$ & $14(36.8)$ & $5(22.7)$ & $12(31.6)$ \\
\hline \multicolumn{5}{|l|}{ Common non-hematologic AEs, $n(\%)$} \\
\hline Diarrhea & $16(18.8)$ & $4(10.5)$ & $3(13.6)$ & $9(36.0)$ \\
\hline Nausea & $8(9.4)$ & 0 & $5(22.7)$ & $3(12.0)$ \\
\hline Vomiting & $8(9.4)$ & 0 & $5(22.7)$ & $3(12.0)$ \\
\hline Constipation & $15(17.6)$ & $8(21.1)$ & $2(9.1)$ & $5(20.0)$ \\
\hline Fatigue & $25(29.4)$ & $11(28.9)$ & $5(22.7)$ & $9(36.0)$ \\
\hline Upper respiratory tract infection & $3(3.5)$ & $1(2.6)$ & 0 & $2(8.0)$ \\
\hline $\begin{array}{l}\text { Rashes or other skin and subcutaneous tissue disorder except } \\
\text { herpes zoster }\end{array}$ & $20(23.5)$ & $16(42.1)$ & $1(4.5)$ & $3(12.0)$ \\
\hline Herpes zoster & $2(2.4)$ & 0 & 0 & $2(8.0)$ \\
\hline Peripheral edema & $11(12.9)$ & $4(10.5)$ & $1(4.5)$ & $6(24.0)$ \\
\hline Pneumonia & $10(11.8)$ & $5(13.2)$ & $2(9.1)$ & $3(12.0)$ \\
\hline Peripheral neuropathy & $5(5.9)$ & $1(2.6)$ & 0 & $4(16.0)$ \\
\hline
\end{tabular}

$A E$, adverse event

${ }^{a}$ This subgroup included Id plus other chemotherapeutics or monoclonal antibody: 11 cases with doxorubicin, 7 with cyclophosphamide, 3 with thalidomide and 1 with daratumumab

that included in the phase 1/2 trial (NCT01217957) [12, 13] (median age 67 versus 66 years), and tended to have more advanced stage diseases (ISS II-III 74\% versus $57 \%, p=$ 0.000 ) and worse ECOG performance status (ECOG PS $>2$ $25 \%$ versus $0 \%, p=0.000$ ) (Table 5). Near half of patients $(45 \%)$ received IRd regimen as in the phase $1 / 2$ clinical (NCT01217957); about $30 \%$ of patients received doublet regimen of ixazomib plus dexamethasone, mostly the elderly (median age 75 years) who might not be able to tolerate triplet regimens; the rest of patients $(25 \%)$ received triplet regimen of Id plus other chemotherapeutics or monoclonal antibody. Yet despite these differences in baseline characteristics and treatment exposure, treatment response and survival outcomes in our study were comparable with the trial (NCT01217957) findings, with an ORR of $95 \%, \geq$ VGPR rate of $66 \%$, and a 12-month PFS of $86 \%$ in our cohort compared with $88 \%$, $58 \%$, and $88 \%$ in the phase $1 / 2$ trial (NCT01217957) [12, 13], respectively (Table 5). Besides, there was no statistically significant difference in treatment response between different ixazomib-based regimens. The results suggest that the ixazomib-based triplet and doublet combination as frontline therapy could be active in a broader real-world population. Of note, despite the promising efficacy results in our whole cohort, we still observed a relatively inferior treatment outcome in those who have high-risk CA and those who were ineligible for clinical trial. Although more than $86 \%$ of the patients with high-risk CA achieved disease response, only one-third achieved a deeper response of $\geq$ VGPR (Table 3). Besides, an inferior PFS was observed in the patients with high-risk $\mathrm{CA}$ and those who were ineligible for clinical trial (Fig. 2 B, F), with a 12month PFS of $76.6 \%$ and $77.4 \%$, respectively, which was lower than the data reported in the NCT01217957 trial $(88 \%)[12,13]$. The possible reasons for these discrepancies may lie in the fact that the patients ineligible for clinical trial were older, with worse ECOG performance status and lower transplantation rate. In fact, patients with age more than 75 years and ECOG PS $>2$ did demonstrate inferior PFS in our cohort (Fig. 2 D, E).

Safety profile was compatible with the previously published phase 1/2 trial (NCT01217957) $[12,13]$. Grade $\geq 3$ AEs were reported in $29 \%$ of the present cohort within follow-up, and $14 \%$ discontinued ixazomib due to AEs in the absence of disease progression. However, the lower grade $\geq 3$ AE rate $(29 \%)$ 
Table 5 Comparison of clinical features and outcomes data from the present study and from the phase $1 / 2$ trial (NCT01217957) of IRd treating newly diagnosed multiple myeloma

\begin{tabular}{llll}
\hline & Present study $(N=85)$ & NCT01217957 trial $(n=65)$ & $P$ \\
\hline Clinical features of patients & & & \\
Median age (range) & $67(35-87)$ & $66(34-86)$ & 0.947 \\
Age $\geq 65$ years, $n(\%)$ & $44(52)$ & $12(18)$ & 0.680 \\
Age $\geq 75$ years, $n(\%)$ & $18(21)$ & & 0.000 \\
ISS stage at diagnosis, $n(\%)$ & & $28(43)$ & \\
I & $22(26)$ & $28(43)$ & 0.000 \\
II & $26(31)$ & $9(14)$ & 0.049 \\
III & $37(43)$ & 0 & 0.263 \\
ECOG performance status, $n(\%)$ & & & \\
$>2$ & $21(25)$ & $56(88)$ & \\
Effectiveness/efficacy & & $37(58)$ & 0.000 \\
ORR $(\geq$ PR), $n(\%)$ & $81(95)$ & 88 & 0.219 \\
$\geq$ VGPR, $n(\%)$ & $56(66)$ & $49(75)$ & $5(8)$ \\
12 -month PFS, $\%$ & 86 & &
\end{tabular}

$I R d$, ixazomib plus lenalidomide and dexamethasone; ISS, International Staging System; ORR, overall response rate; $V G P R$, very good partial response; $P F S$, progression-free survival; $A E$, adverse event reported here is likely to be attributed to a reporting bias due to the retrospective nature of this study. Despite the potential bias, these findings still reflected that ixazomib-based regimens are well-tolerated. Of note, no grade $>2 \mathrm{PN}$ was observed in our cohort. This finding was encouraging, especially in the consideration that PN has been one of the major concerns when using proteasome inhibitor and the reported incidence of bortezomibinduced PN was 40\% [25]. Despite the lower $>3 \mathrm{AE}$ rate and high response rate in our cohort, we observed high discontinuation rate $(14 \%$ vs. $8 \%)$ in our data. Unlike in strictly controlled and well-managed clinical trials, the treatment endurance may be rather low in real-life practice, since physicians may be less motivated to encourage their patients on the need to continue treatment in routine practice, and patients in real-life may tend to give up a therapy despite few side effects.

In our real-world cohort, ten patients received single-agent ixazomib maintenance after induction. None of the patients discontinued ixazomib maintenance due to AEs, and all but the one who had disease progression remained on maintenance therapy until the date of the last follow-up. The favorable tolerability observed in our cohort highlighted the feasibility of maintenance therapy with ixazomib. The SCT rate in our cohort was only $15.4 \%$ in patients who were evaluated as transplantationeligible (ages 18-64 years, ECOG 0-1, without severe comorbidities). This data was quite consistent with the reported SCT rate $(14.4 \%)$ in another large real-world study of 940 Chinese NDMM patients [26], and it did reflect the real situation in China. The possible reasons for the low SCT rates include fears for transplant, financial concerns (many fees for SCT, including melphalan, were not covered by health insurance), limited drug accessibility (melphalan has just commercially entered China in 2019), shortage of qualified medical service (only large reference centers or specialized centers have the ability to perform SCT), and disturbance on routine medical service during COVID.

The ixazomib-based all-oral regimens, including IRd, ICd, and ITd, have demonstrated promising efficacy, safety, and tolerability in rigorously controlled clinical trials for NDMM [12-15, 27, $28]$. Besides the greater convenience brought by the oral administration, the NDMM patients who received all-oral regimens have been shown to have lower economic burden of illness, less activity impairment, and lower productivity loss [29]. These facts together explained the reason for choosing ixazomib-based regimens as frontline therapy in selected real-world patients. More importantly, all-oral therapy may be able to help in decreasing the exposure to and infection with COVID-19 [16].

Indeed, real-world data are emerging for the use of ixazomib in RRMM, but such data in the frontline treatment setting with ixazomib are still limited. The findings in the present study are encouraging. Although the follow-up was fairly short (median 10.3 months), as ixazomib was just introduced in China in April 2018, our real-world data did demonstrate that the ixazomib-based regimens were active in routine practice for NDMM. On the basis of this analysis and the other co-factors that should be taken into consideration when making treatment decisions NDMM patients in routine practice, ixazomib-based regimen would be an optimal alternative as frontline therapy.

In conclusion, herein we reported the data from the first real-life, multi-center study on the effectiveness and safety profile of ixazomib-based frontline therapy in NDMM. 
Authorship contributions J.L., L.B., Z.-J.X, S.-L.W., X.Z., K.Y.D., W.-H.Z., W.Y., B.-Z.L, C.-C.F., B.C., L.-M.H., L.W., J.L., Y.Y., T.-H.X., W.-D.W., Y.H., and G.-L.W. collected data; J.L. analyzed results and created the figures; L.B., Z.J.X, and P.L. designed the research; J.L. drafted the manuscript; and L.B., Z.-J.X, and P.L. gave the final approval of the paper.

Funding This study has been partially supported by the National Key New Drug Creation Special Programs (2017ZX09304-021).

Data availability The data that support the findings of this study are available from the corresponding author upon reasonable request.

\section{Compliance with ethical standards}

Conflict of interest The authors declare that they have no conflict of interest.

Ethics approval The study was approved by the institutional ethics committee (B2019-228R) and conducted in accordance with the 1964 Helsinki declaration and its later amendments.

Consent to participate Informed consent for participation was obtained from all enrolled individuals.

Consent for publication Informed consent for the publication of data was obtained from all enrolled individuals.

Open Access This article is licensed under a Creative Commons Attribution 4.0 International License, which permits use, sharing, adaptation, distribution and reproduction in any medium or format, as long as you give appropriate credit to the original author(s) and the source, provide a link to the Creative Commons licence, and indicate if changes were made. The images or other third party material in this article are included in the article's Creative Commons licence, unless indicated otherwise in a credit line to the material. If material is not included in the article's Creative Commons licence and your intended use is not permitted by statutory regulation or exceeds the permitted use, you will need to obtain permission directly from the copyright holder. To view a copy of this licence, visit http://creativecommons.org/licenses/by/4.0/.

\section{References}

1. Kumar SK, Dispenzieri A, Lacy MQ, Gertz MA, Buadi FK, Pandey S, Kapoor P, Dingli D, Hayman SR, Leung N, Lust J, McCurdy A, Russell SJ, Zeldenrust SR, Kyle RA, Rajkumar SV (2014) Continued improvement in survival in multiple myeloma: changes in early mortality and outcomes in older patients. Leukemia 28(5): 1122-1128. https://doi.org/10.1038/leu.2013.313

2. Rajkumar SV (2019) Multiple myeloma: every year a new standard? Hematol Oncol 37(Suppl 1):62-65. https://doi.org/10.1002/ hon. 2586

3. Attal M, Lauwers-Cances V, Hulin C, Leleu X, Caillot D, Escoffre M, Arnulf B, Macro M, Belhadj K, Garderet L, Roussel M, Payen C, Mathiot C, Fermand JP, Meuleman N, Rollet S, Maglio ME, Zeytoonjian AA, Weller EA, Munshi N, Anderson KC, Richardson PG, Facon T, Avet-Loiseau H, Harousseau JL, Moreau P, Study IFM (2017) Lenalidomide, bortezomib, and dexamethasone with transplantation for myeloma. N Engl J Med 376(14):1311-1320. https://doi.org/10.1056/NEJMoa1611750
4. Durie BGM, Hoering A, Abidi MH, Rajkumar SV, Epstein J, Kahanic SP, Thakuri M, Reu F, Reynolds CM, Sexton R, Orlowski RZ, Barlogie B, Dispenzieri A (2017) Bortezomib with lenalidomide and dexamethasone versus lenalidomide and dexamethasone alone in patients with newly diagnosed myeloma without intent for immediate autologous stem-cell transplant (SWOG S0777): a randomised, open-label, phase 3 trial. Lancet 389(10068):519-527. https://doi. org/10.1016/S0140-6736(16)31594-X

5. Mateos MV, Cavo M, Blade J, Dimopoulos MA, Suzuki K, Jakubowiak A, Knop S, Doyen C, Lucio P, Nagy Z, Pour L, Cook M, Grosicki S, Crepaldi A, Liberati AM, Campbell P, Shelekhova T, Yoon SS, Iosava G, Fujisaki T, Garg M, Krevvata M, Chen Y, Wang J, Kudva A, Ukropec J, Wroblewski S, Qi M, Kobos R, San-Miguel J (2020) Overall survival with daratumumab, bortezomib, melphalan, and prednisone in newly diagnosed multiple myeloma (ALCYONE): a randomised, open-label, phase 3 trial. Lancet 395(10218):132-141. https://doi.org/10.1016/S0140-6736(19)32956-3

6. Ludwig H, Durie BG, McCarthy P, Palumbo A, San Miguel J, Barlogie B, Morgan G, Sonneveld P, Spencer A, Andersen KC, Facon T, Stewart KA, Einsele H, Mateos MV, Wijermans P, Waage A, Beksac M, Richardson PG, Hulin C, Niesvizky R, Lokhorst H, Landgren O, Bergsagel PL, Orlowski R, Hinke A, Cavo M, Attal M, International Myeloma Working G (2012) IMWG consensus on maintenance therapy in multiple myeloma. Blood 119(13):3003-3015. https://doi.org/10.1182/blood-2011-11-374249

7. Palumbo A, Gay F, Cavallo F, Di Raimondo F, Larocca A, Hardan I, Nagler A, Petrucci MT, Hajek R, Pezzatti S, Delforge M, Patriarca F, Donato F, Cerrato C, Nozzoli C, Yu Z, Boccadifuoco L, Caravita T, Benevolo G, Guglielmelli T, Vincelli D, Jacques C, Dimopoulos MA, Ciccone G, Musto P, Corradini P, Cavo M, Boccadoro M (2015) Continuous therapy versus fixed duration of therapy in patients with newly diagnosed multiple myeloma. J Clin Oncol 33(30):3459-3466. https://doi.org/10.1200/JCO.2014.60.2466

8. Yong K, Delforge M, Driessen C, Fink L, Flinois A, Gonzalez-McQuire S, Safaei R, Karlin L, Mateos MV, Raab MS, Schoen P, Cavo M (2016) Multiple myeloma: patient outcomes in real-world practice. Br J Haematol 175(2):252-264. https://doi.org/10.1111/bjh.14213

9. Hou J, Jin J, Xu Y, Wu D, Ke X, Zhou D, Lu J, Du X, Chen X, Li J, Liu J, Gupta N, Hanley MJ, Li H, Hua Z, Wang B, Zhang X, Wang H, van de Velde H, Richardson PG, Moreau P (2017) Randomized, double-blind, placebo-controlled phase III study of ixazomib plus lenalidomide-dexamethasone in patients with relapsed/refractory multiple myeloma: China Continuation study. J Hematol Oncol 10(1):137. https://doi.org/10.1186/s13045-017-0501-4

10. Moreau P, Masszi T, Grzasko N, Bahlis NJ, Hansson M, Pour L, Sandhu I, Ganly P, Baker BW, Jackson SR, Stoppa AM, Simpson DR, Gimsing P, Palumbo A, Garderet L, Cavo M, Kumar S, Touzeau C, Buadi FK, Laubach JP, Berg DT, Lin J, Di Bacco A, Hui AM, van de Velde H, Richardson PG, Group T-MS (2016) Oral ixazomib, lenalidomide, and dexamethasone for multiple myeloma. $\mathrm{N}$ Engl $\mathrm{J}$ Med 374(17):1621-1634. https://doi.org/10.1056/NEJMoa1516282

11. Terpos E, Ramasamy K, Maouche N, Minarik J, NtanasisStathopoulos I, Katodritou E, Jenner MW, Plonkova H, Gavriatopoulou M, Vallance GD, Pika T, Kotsopoulou M, Kothari J, Jelinek T, Kastritis E, Aitchison R, Dimopoulos MA, Zomas A, Hajek R (2020) Real-world effectiveness and safety of ixazomib-lenalidomide-dexamethasone in relapsed/refractory multiple myeloma. Ann Hematol 99(5):1049-1061. https://doi.org/10. 1007/s00277-020-03981-z

12. Kumar SK, Berdeja JG, Niesvizky R, Lonial S, Laubach JP, Hamadani M, Stewart AK, Hari P, Roy V, Vescio R, Kaufman JL, Berg D, Liao E, Di Bacco A, Estevam J, Gupta N, Hui AM, Rajkumar V, Richardson PG (2014) Safety and tolerability of ixazomib, an oral proteasome inhibitor, in combination with lenalidomide and dexamethasone in patients with previously 
untreated multiple myeloma: an open-label phase 1/2 study. Lancet Oncol 15(13):1503-1512. https://doi.org/10.1016/S1470-2045(14) 71125-8

13. Kumar SK, Berdeja JG, Niesvizky R, Lonial S, Laubach JP, Hamadani M, Stewart AK, Hari P, Roy V, Vescio R, Kaufman JL, Berg D, Liao E, Rajkumar SV, Richardson PG (2019) Ixazomib, lenalidomide, and dexamethasone in patients with newly diagnosed multiple myeloma: long-term follow-up including ixazomib maintenance. Leukemia 33(7):1736-1746. https://doi. org/10.1038/s41375-019-0384-1

14. Dimopoulos MA, Grosicki S, Jedrzejczak WW, Nahi H, Gruber A, Hansson M, Gupta N, Byrne C, Labotka R, Teng Z, Yang H, Grzasko N, Kumar S (2019) All-oral ixazomib, cyclophosphamide, and dexamethasone for transplant-ineligible patients with newly diagnosed multiple myeloma. Eur J Cancer 106:89-98. https:// doi.org/10.1016/j.ejca.2018.09.011

15. Kumar SK, Buadi FK, LaPlant B, Halvorson A, Leung N, Kapoor P, Dingli D, Gertz MA, Go RS, Bergsagel PL, Lin Y, Dispenzieri A, Hwa YL, Fonder A, Hobbs M, Fonseca R, Hayman SR, Stewart AK, Lust JA, Mikhael J, Gonsalves W, Reeder C, Skacel T, Rajkumar SV, Lacy MQ (2018) Phase 1/2 trial of ixazomib, cyclophosphamide and dexamethasone in patients with previously untreated symptomatic multiple myeloma. Blood Cancer J 8(8):70. https://doi.org/10.1038/s41408-018-0106-3

16. Al Saleh AS, Sher T, Gertz MA (2020) Multiple myeloma in the time of COVID-19. Acta Haematol:1-7. https://doi.org/10.1159/ 000507690

17. Mian H, Grant SJ, Engelhardt M, Pawlyn C, Bringhen S, Zweegman S, Stege CAM, Rosko AE, von Lilienfeld-Toal M, Wildes TM (2020) Caring for older adults with multiple myeloma during the COVID-19 pandemic: perspective from the International Forum for Optimizing Care of Older Adults with Myeloma. J Geriatr Oncol 11(5):764-768. https://doi.org/10. 1016/j.jgo.2020.04.008

18. Terpos E, Engelhardt M, Cook G, Gay F, Mateos MV, NtanasisStathopoulos I, van de Donk N, Avet-Loiseau H, Hajek R, Vangsted AJ, Ludwig H, Zweegman S, Moreau P, Einsele H, Boccadoro M, San Miguel J, Dimopoulos MA, Sonneveld P (2020) Management of patients with multiple myeloma in the era of COVID-19 pandemic: a consensus paper from the European Myeloma Network (EMN). Leukemia. 34:2000-2011. https://doi. org/10.1038/s41375-020-0876-Z

19. Engelhardt M, Shoumariyeh K, Rosner A, Ihorst G, Biavasco F, Meckel K, von Metzler I, Treurich S, Hebart H, Grube M, Kull M, Bassermann F, Schafer-Eckart K, Hoferer A, Einsele H, Rasche L, Wasch R (2020) Clinical characteristics and outcome of multiple myeloma patients with concomitant COVID-19 at Comprehensive Cancer Centers in Germany. Haematologica.:haematol.2020.262758. https://doi.org/10.3324/haematol.2020.262758

20. Bergin K, McQuilten Z, Moore E, Wood E, Spencer A (2017) Myeloma in the real world: what is really happening? Clin Lymphoma Myeloma Leuk 17(3):133-144 e131. https://doi.org/ 10.1016/j.clml.2016.12.002

21. Shah JJ, Abonour R, Gasparetto C, Hardin JW, Toomey K, Narang M, Srinivasan S, Kitali A, Zafar F, Flick ED, Rifkin RM (2017) Analysis of common eligibility criteria of randomized controlled trials in newly diagnosed multiple myeloma patients and extrapolating outcomes. Clin Lymphoma Myeloma Leuk 17(9):575-583 e572. https://doi.org/10.1016/j.clml.2017.06.013

22. Rajkumar SV, Dimopoulos MA, Palumbo A, Blade J, Merlini G, Mateos MV, Kumar S, Hillengass J, Kastritis E, Richardson P, Landgren O, Paiva B, Dispenzieri A, Weiss B, LeLeu X, Zweegman S, Lonial S, Rosinol L, Zamagni E, Jagannath S, Sezer O, Kristinsson
SY, Caers J, Usmani SZ, Lahuerta JJ, Johnsen HE, Beksac M, Cavo M, Goldschmidt H, Terpos E, Kyle RA, Anderson KC, Durie BG, Miguel JF (2014) International Myeloma Working Group updated criteria for the diagnosis of multiple myeloma. Lancet Oncol 15(12): e538-e548. https://doi.org/10.1016/S1470-2045(14)70442-5

23. Ross FM, Avet-Loiseau H, Ameye G, Gutierrez NC, Liebisch P, O'Connor S, Dalva K, Fabris S, Testi AM, Jarosova M, Hodkinson C, Collin A, Kerndrup G, Kuglik P, Ladon D, Bernasconi P, Maes B, Zemanova Z, Michalova K, Michau L, Neben K, Hermansen NE, Rack K, Rocci A, Protheroe R, Chiecchio L, Poirel HA, Sonneveld P, Nyegaard M, Johnsen HE, European Myeloma N (2012) Report from the European Myeloma Network on interphase FISH in multiple myeloma and related disorders. Haematologica 97(8):1272-1277. https://doi.org/10.3324/haematol.2011.056176

24. Kumar S, Paiva B, Anderson KC, Durie B, Landgren O, Moreau P, Munshi N, Lonial S, Blade J, Mateos MV, Dimopoulos M, Kastritis E, Boccadoro M, Orlowski R, Goldschmidt H, Spencer A, Hou J, Chng WJ, Usmani SZ, Zamagni E, Shimizu K, Jagannath S, Johnsen HE, Terpos E, Reiman A, Kyle RA, Sonneveld P, Richardson PG, McCarthy P, Ludwig H, Chen W, Cavo M, Harousseau JL, Lentzsch S, Hillengass J, Palumbo A, Orfao A, Rajkumar SV, Miguel JS, AvetLoiseau H (2016) International Myeloma Working Group consensus criteria for response and minimal residual disease assessment in multiple myeloma. Lancet Oncol 17(8):e328-e346. https://doi.org/10.1016/ S1470-2045(16)30206-6

25. Morawska M, Grzasko N, Kostyra M, Wojciechowicz J, Hus M (2015) Therapy-related peripheral neuropathy in multiple myeloma patients. Hematol Oncol 33(4):113-119. https://doi.org/10.1002/hon.2149

26. Lu J, Lu J, Chen W, Huo Y, Huang X, Hou J, Chinese Medical Doctor Association Hematology B (2014) Clinical features and treatment outcome in newly diagnosed Chinese patients with multiple myeloma: results of a multicenter analysis. Blood Cancer J 4: e239. https://doi.org/10.1038/bcj.2014.55

27. Dimopoulos MA, Gay F, Schjesvold F, Beksac M, Hajek R, Weisel KC, Goldschmidt H, Maisnar V, Moreau P, Min CK, Pluta A, Chng WJ, Kaiser M, Zweegman S, Mateos MV, Spencer A, Iida S, Morgan G, Suryanarayan K, Teng Z, Skacel T, Palumbo A, Dash AB, Gupta N, Labotka R, Rajkumar SV, group T-Ms (2019) Oral ixazomib maintenance following autologous stem cell transplantation (TOURMALINE-MM3): a double-blind, randomised, placebo-controlled phase 3 trial. Lancet 393(10168):253-264. https://doi.org/10.1016/S0140-6736(18)33003-4

28. Zweegman S, Stege CAM, Haukas E, Schjesvold FH, Levin MD, Waage A, Leys RBL, Klein SK, Szatkowski D, Axelsson P, Hieu Do T, Knut-Bojanowska D, van der Spek E, Svirskaite A, Klostergaard A, Salomo M, Blimark C, Ypma PF, Mellqvist UH, Poddighe PJ, Stevens-Kroef M, van de Donk N, Sonneveld P, Hansson M, van der Holt B, Abildgaard N (2020) Ixazomibthalidomide-low dose dexamethasone induction followed by maintenance therapy with ixazomib or placebo in newly diagnosed multiple myeloma patients not eligible for autologous stem cell transplantation; results from the randomized phase II HOVON-126/NMSG 21.13 trial. Haematologica. doi:https://doi.org/10.3324/haematol.2019.240374

29. Merola D, Yong C, Noga SJ, Shermock KM (2018) Costs associated with productivity loss among U.S. patients newly diagnosed with multiple myeloma receiving oral versus injectable chemotherapy. J Manag Care Spec Pharm 24(10):1019-1026. https://doi.org/ 10.18553/jmcp.2018.24.10.1019

Publisher's note Springer Nature remains neutral with regard to jurisdictional claims in published maps and institutional affiliations. 